\title{
Bridging the gap between the emergency department and outpatient care: feasibility of a short-term psychiatric crisis intervention for children and adolescents
}

\author{
Idit Dekel ${ }^{1,2} \cdot$ Nimrod Hertz-Palmor $^{1,3}$ (1) Shirel Dorman-Ilan ${ }^{1} \cdot$ Mor Reich-Dvori ${ }^{1} \cdot$ Doron Gothelf ${ }^{1,2,4}$. \\ Itai M. Pessach ${ }^{1,2}$
}

Received: 16 June 2021 / Accepted: 29 September 2021 / Published online: 26 October 2021

(c) The Author(s), under exclusive licence to Springer-Verlag GmbH Germany 2021

\begin{abstract}
Child and adolescent mental health systems are facing limited resources of available psychosocial interventions, often leading to long waiting lists for acceptance to treatment. We describe the feasibility of a short-term ( $8-10$ sessions) psychological crisis intervention (CI) protocol for children and adolescents aged 8-17 years $(n=30$, mean \pm standard deviation $12.9 \pm 2.4$ years) who were referred to an outpatient mental health clinic due to suicidal ideation, aggression, severe anxiety, or extreme family conflict. The participants were assessed before and after the CI, and at a 3-6-months follow-up visit. The psychiatric assessments included clinical evaluation by a senior psychiatrist, and the completion of self-report questionnaires by both the participants and their parents. Following the establishment of the $\mathrm{CI}$ unit, the waiting lists for urgent cases were reduced from a median of 84 days in the two preceding years to 23 days in the following 3 years $(H[2]=18.5, p<0.0001)$ for patients of the CI unit. A 1-year psychiatric follow-up after the end of the CI revealed that $72 \%$ did not require additional psychotherapy. The overall clinical evaluation measures (clinical evaluation, parents-report and child report) improved and had been preserved at the 3-6-months follow-up. Our results demonstrate the feasibility of a short-term CI protocol for expediting admission to treatment for urgent psychiatric cases.
\end{abstract}

Keywords Children $\cdot$ Adolescents $\cdot$ Crisis intervention $\cdot$ Waiting list $\cdot$ Short-term psychotherapy $\cdot$ Suicide prevention

\section{Introduction}

One of the main challenges faced by child and adolescent mental health systems is the limited resources of available psycho-social interventions [1, 2]. In recent years, the number of emergency department (ED) visits for psychiatric illness has been rising worldwide [1, 3, 4]. Part of this rise

Idit Dekel

dekel.idit@gmail.com

1 The Child Psychiatry Division, Edmond and Lily Safra Children's Hospital, Sheba Medical Center, Tel Hashomer, Ramat Gan, Israel

2 Sackler Faculty of Medicine, Tel Aviv University, Tel Aviv, Israel

3 School of Psychological Sciences, Tel Aviv University, Tel Aviv, Israel

4 Sagol School of Neuroscience, Tel Aviv University, Tel Aviv, Israel includes youths with common psychiatric crises, such as suicidal ideation, aggression, severe anxiety, or extreme family conflict, that are referred urgently to emergency departments (ED) or to psychiatric clinics, where they are invariably confronted by very long waiting-lists for intervention [5]. A "mental health crisis" is a broad term used to describe a variety of psychiatric and psychological conditions, and is defined as "an acute disturbance of thought, mood, or behavior, that requires immediate intervention and the resources available to manage the situation are not available at the time and place of occurrence" [6]. Community-based models have recently been described for the care of youth in mental health crisis that present to the ED [7]. Similar to the world trend, a rise in psychiatric emergencies, such as suicidal behaviors, presenting to pediatric EDs, is well documented in Israel [8]. However, community-based services are scarce. Since the reform in Israeli mental health services in 2015 , all psychiatric services are now provided exclusively by Health Maintenance Organizations (HMOs). The majority of these services are provided in outpatient clinic settings that are 
overloaded. In case of psychiatric emergency patients are referred to EDs. At the Edmond and Lily Safra Children's Hospital-Sheba Medical Center-Tel Hashomer, a tertiary pediatric hospital in Israel, the annual number of psychiatric emergencies presenting to the ED increased in recent years and now stands at about 350 cases annually. This increase has led to an overload of the psychiatric outpatient clinic at our hospital, with an average of a 6 months wait time to treatment overall, and about 4 months for urgent cases referred from the ED.

In this study we describe the feasibility of a psychiatric crisis intervention (CI) model aimed at offering immediate psychiatric evaluation and treatment with short-term psychotherapy for diverse common psychiatric emergencies in an outpatient psychiatric clinic setting. The main objectives were to reduce waiting time for urgent treatment as well as to evaluate treatment dropout rates and the need for additional treatment during the first year following CI. We employed standard clinical assessments to measure clinical improvement. We hypothesized that a short (8-10 sessions) psychotherapeutic intervention will result in improvement of psychiatric symptoms that will be maintained at a 3-6-months follow-up, and that the instigation of this protocol will lead to a reduction in waiting time for therapy for urgent cases.

\section{Methods}

\section{Population}

Cases referred to a child psychiatry clinic at a tertiary medical center in Israel during 2016-2019 were classified as urgent and referred to the CI unit according to the following criteria: 1. Clinical Global Impression Inventory-Severity scale (CGI-S) $\geq 4 ; 2$. Children's Global Assessment Scale $(\mathrm{CGAS}) \leq 60 ; 3$. at least one of the following: suicidal risk, defined as a recent suicide attempt during the last month; Columbia-Suicide Severity Rating Scale (C-SSRS) $\geq 3$; violent outbursts and severe oppositional and defiant behavior, severe anxiety, obsessive-compulsive symptoms, or major life events (e.g., recent divorce, physical illness, loss of a family member) leading to a severe adjustment disorder.

During 2016-2019, approximately 120 cases were referred to the initial psychiatric evaluation. Cases with mild psychopathology (CGI-S $<4$ at the time of initial assessment) were deferred to routine psychiatric follow-up or other interventions in the clinic and were excluded from the CI and from this study. In the majority of the remaining cases not treated in the CI unit, the parents were not interested in the intervention offered. Less common reasons for exclusion were cases of eating disorders that were referred to a specialized unit at our center.

\section{Procedure}

Children and adolescents who met the inclusion criteria were referred to the CI unit in the outpatient clinic at Safra Children's Hospital after an initial psychiatric evaluation by a senior child psychiatrist. We developed four brief intervention protocols based upon principles from well-established types of psychotherapy: 1) cognitive-behavioral therapy (CBT) for adolescents with anxiety disorders or obsessive-compulsive disorder, and parent training based upon the Supportive parenting for anxious childhood emotions (SPACE) protocol for anxious children under 12 years; 2) CBT-suicide prevention for suicidality; 3) parents training for aggression, violent outbursts, or school refusal based upon non-violent resistance treatment; and 4) time-limited psychotherapy for adolescents based upon James Mann's time-limited psychotherapy principles for adolescents facing major life events leading to severe adjustment disorders [9-14]. A multi-disciplinary team, consisting of a senior child psychiatrist, social workers and a psychologist, planned an 8-session intervention for each case after the initial psychiatric assessment. A focus for intervention was defined based on the information collected in the initial assessment, and the treatment protocol was selected accordingly. The focus of intervention was communicated to the patient and family in the first therapy session. In addition, psychiatric follow-up visits were scheduled as needed: in cases treated with pharmacotherapy and/or in cases with suicidal behaviors, psychiatric visits were scheduled in 2-4-week intervals. In other cases, psychiatric follow-up was conducted at the termination of the psychotherapeutic intervention, and in 3-months intervals thereafter.

\section{Measures}

In this study we used the self- and parent-report questionnaires Children's Depression Inventory (CDI) and State-Trait Anxiety Inventory (STAI) [15, 16]. A senior child psychiatrist completed the Clinical Global Impression Scale, rating the severity of the main diagnosis (CGIS), the Children's Global Assessment of functioning Scale (CGAS), and the Columbia-Suicide Severity Rating Scale (C-SSRS) [17-19]. The participants were assessed pre- and post-treatment, and at a 3-6-months follow-up visit since the final session.

\section{Data analysis}

Kruskal-Wallis test was conducted to compare the time on waiting list before and after the implementation of the CI unit (both for patients who admitted to the unit, and patients 
who declined CI treatment). Linear mixed-effects models were applied to compare pre-therapy (as reference), posttherapy, and follow-up clinical reports, including clinician assessments (CGI-S, CGAS, C-SSRS), parents' reports (CDI, STAI-State, STAI-Trait), and participants' self-report (CDI, STAI-C Trait). We controlled for interpersonal variance and missing data by modelling the participants as random factors, and compared baseline differences between participants with full and missing data to assure that our results were not affected by survivorship bias. Baseline differences were tested using the non-parametric Mann-Whitney test, due to the small number of participants on each group. We set the significance level at $\alpha<0.05$. Analyses were conducted with R's-lmerTest package [20].

\section{Results}

\section{Participants}

Fifty-three patients (29 girls, 54.7\%) were treated in the CI unit between 2016 and 2019. Twenty-three were referred directly from the ED $(43 \%)$. The final analytic sample included 30 patients (mean age \pm standard deviation $=13.1 \pm 2.4,20$ girls [66.7\%]) who completed baseline and endpoint assessments (Table 1). Seventeen patients completed follow-up assessments (Fig. 1). Of the 30 patients that were included in the analysis, 6 (20.0\%) were on psychopharmacological treatment prior to their referral to the unit, while $14(46.7 \%)$ additional patients were prescribed psychiatric medications during the $\mathrm{CI}$ treatment. All patients adhered to their medications until the end of the intervention, and $4(20.0 \%)$ discontinued pharmacotherapy during the first 6 months following the treatment endpoint (see Table S1 in the supplementary materials for detailed description of pharmacotherapy during the intervention).

\section{Time on waiting list}

The median time on waiting list among referrals from the ED was 84 days prior to the establishment of the CI unit (2014-2015). This time was significantly shortened after the CI unit was establishment (2016-2019) with a median of 23 days for patients that were treated by the unit, and 52 days for patients who declined CI treatment. Group differences were significant $(H[2]=18.5, p<0.0001$, $\left.\eta^{2}=0.19\right)$. Post-hoc comparisons using Mann-Whitney test (corrected for multiple comparisons with FDR correction [21]) revealed that the time on waiting list among CI patients was significantly shorter compared with the waiting list time of patients treated prior to the unit's establishment (during 2014-2015) $(U=854, p<0.001)$ as well as the waiting list time of patients who declined
Table 1 Sociodemographic characteristics of the study sample

\begin{tabular}{ll}
\hline Characteristics & \\
\hline$n(\%)$ & 30 \\
Age, mean \pm SD (range) & $12.9 \pm 2.4(8-17)$ \\
Male/female, $n(\%)$ & $10 / 20(33.3 \% / 66.7 \%)$ \\
Parents training only & $16(53.3 \%)$ \\
Parents training + child therapy & $14(46.7 \%)$ \\
Main problem (may be multiple) & \\
School attendance refusal & $9(30.0 \%)$ \\
Suicidal ideation/attempt, self-harm & $9(30.0 \%)$ \\
Violent outbursts & $8(26.7 \%)$ \\
Anxiety & $3(10.0 \%)$ \\
Major life events & $2(6.7 \%)$ \\
Psychiatric diagnoses & \\
Adjustment disorder & $12(40.0 \%)$ \\
Oppositional defiant disorder, conduct & $10(33.3 \%)$ \\
$\quad$ disorder & \\
Major depression, dysthymia & $9(30.0 \%)$ \\
Attention deficit/hyperactive disorder & $7(23.3 \%)$ \\
Generalized anxiety disorder & $5(16.7 \%)$ \\
Social anxiety disorder & $2(6.7 \%)$ \\
Panic disorder & $1(3.3 \%)$ \\
Post-traumatic stress disorder & $1(3.3 \%)$ \\
Dissociative and conversion disorders & $1(3.3 \%)$ \\
Gender dysphoria & $1(3.3 \%)$ \\
Medications & \\
Antidepressants & $14(46.7 \%)$ \\
Antipsychotics & $11(36.7 \%)$ \\
Stimulants & $7(23.3 \%)$ \\
Benzodiazepines & $1(3.3 \%)$ \\
None & $7(23.3 \%)$ \\
\hline &
\end{tabular}

CI treatment ( $U=586.5, p=0.005)$. The time on waiting list for patients who declined CI treatment was similar to that of patients treated prior to the CI unit establishment $(U=172.5, p=0.64)$. The distribution of time on waiting list before and after the implementation of the CI unit is depicted in Fig. 2.

There were no baseline differences in any of the outcome measures between participants who completed all three timepoints $(n=17)$ and participants who participated in baseline and post assessments but did not complete followup $(n=13)$.

\section{Need for post-Cl treatment}

At the 1-year post-intervention follow-up, two patients (3.8\%) required admission to a closed psychiatric ward, two (3.8\%) were referred to a psychiatric daycare service, and 38 (72\%) did not require any additional psychotherapy. These 


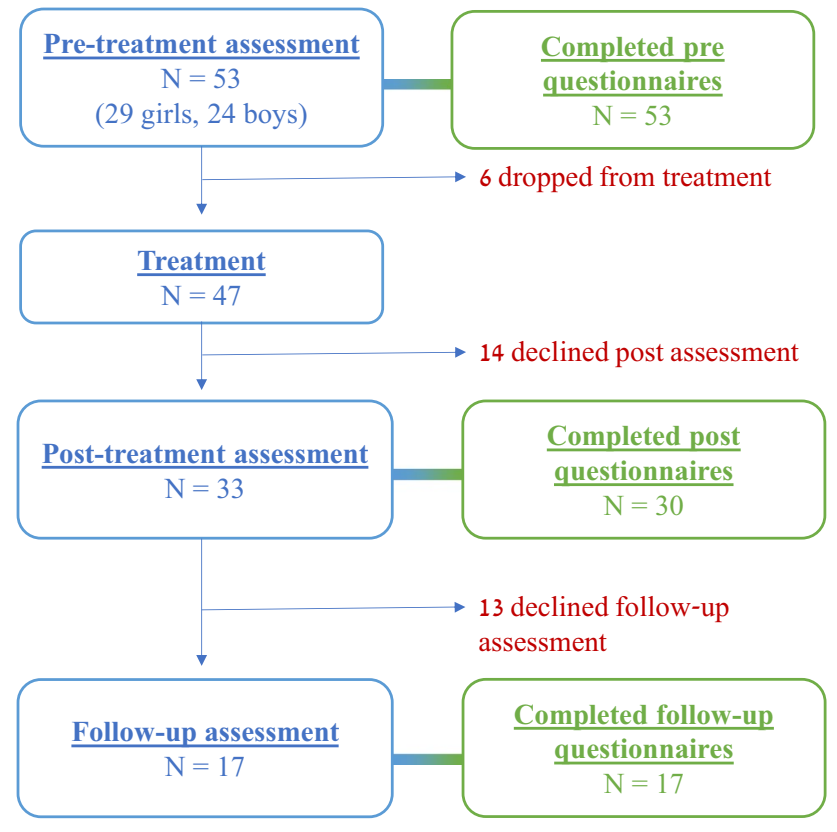

Fig. 1 Consort diagram for the crisis intervention patients. We enrolled 53 patients and acquired complete data for 30 patients

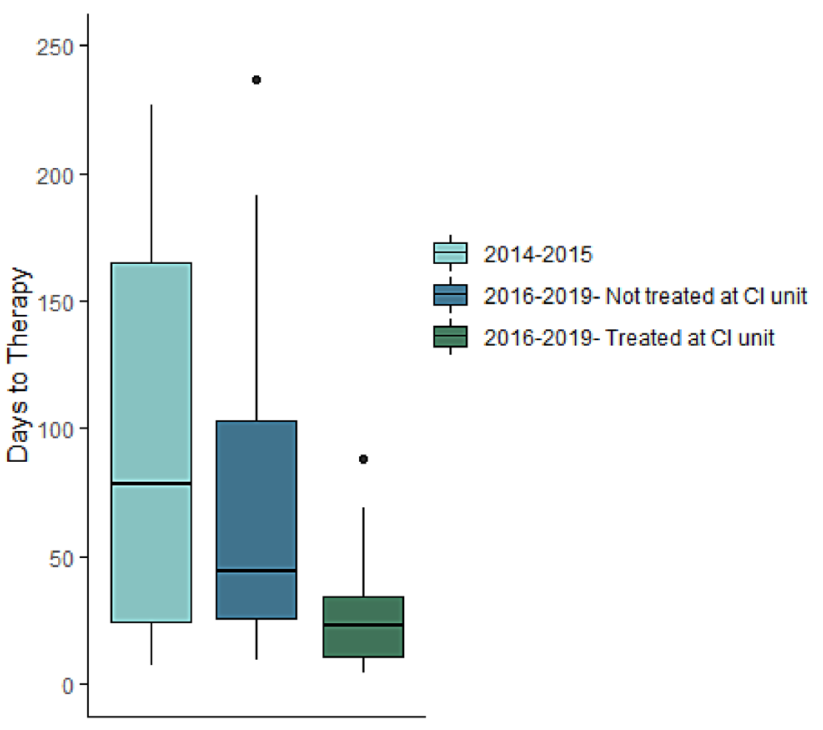

Fig. 2 Time on waiting list for patients who were referred from the ED prior to the establishment of the CI unit (2014-2015, Median $=84$ days), patients who were treated by the unit (2016-2019, $\mathrm{Md}=23$ ) and patients who referred from the ED between 2016 and 2019 but declined CI treatment $(\mathrm{Md}=52)$. Time on waiting list for CI treatment was significantly shorter for CI treatment, compared to waiting time in 2014-2015 $(p<0.001)$, but was no different for patients who declined CI treatment $(p=0.64)$

data were collected from the electronic medical records of all patients that completed the $\mathrm{CI}$ intervention, including those that did not complete the research questionnaires $(N=53)$.

\section{Clinician assessments}

There was a significant improvement between pre- and post-interventions in CGI-S (pre-CI $=4.9$, post-CI $=3.5$; $p<0.0001$ ) and CGAS (pre-CI $=41.2$, post-CI $=52.3$; $p<0.0001)$ scores. The improvement was preserved at the 3-6-months follow-up (Table 2).

\section{Parental reports}

The parental reports showed a significant decrease in the participants' depressive symptoms (CDI; pre-CI $=22.2$, post-CI $=16.8 ; p<0.0001)$ and state anxiety (STAI-S; pre$\mathrm{CI}=48.6$, post-CI $=40.0 ; p=0.001$ ), with no change in trait anxiety symptoms. Improvement was preserved at follow-up (Table 2).

\section{Participants' self-report}

The participants' self-reports showed a significant decrease in depressive and anxiety symptoms between pre- and postCI (CDI: pre $=22.5$, post $=18.2, p=0.042$; STAI-C: pre$\mathrm{CI}=44.0$, post-CI $=40.8, p=0.024)$. Improvement in those traits was not preserved at follow-up (Table 2).

\section{Discussion}

As urgent pediatric mental health issues and subsequently pediatric ED visits for mental health problems are on the rise worldwide, healthcare systems struggle to meet the increasing demand for pediatric psychiatric services [1]. In many cases, pediatric psychiatric outpatient resources are limited and as a result, urgent cases face exceedingly long waiting lists for intervention, leaving children in acute crisis untreated for long periods of time. This phenomenon is becoming a real public health concern, leading to a rise in morbidity, increase in complexity and eventually requiring longer intervention and even more resources $[1,22]$. Therefore, brief interventions aimed at stabilizing and reducing risk in these acute situations are crucial. The findings of this study demonstrate the feasibility and utility of a novel psychiatric CI model for children and adolescents. To the best of our knowledge, this is the first model that offers short-term intervention for diverse common psychiatric emergencies in the setting of a child and adolescent psychiatric outpatient clinic. Our intervention integrated principles from wellestablished treatment protocols, and dramatically reduced the waitlist time for treatment of acute cases. Furthermore, dropout rates were low, and most of the patients (72\%) did 
Table 2 Comparisons of clinician-rated and parent and participant-reported measures between pre-treatment, post-treatment, and follow-up assessments

\begin{tabular}{|c|c|c|c|c|c|c|c|c|}
\hline \multirow[t]{2}{*}{ MEASURE } & \multirow[t]{2}{*}{ Observations } & \multirow{2}{*}{$\begin{array}{l}\text { Pre } \\
\text { M (SD) }\end{array}$} & \multicolumn{3}{|l|}{ Post } & \multicolumn{3}{|l|}{ Follow-up } \\
\hline & & & $\mathrm{M}(\mathrm{SD})$ & $\begin{array}{l}\text { Non- } \\
\text { standard- } \\
\text { ized B }\end{array}$ & $p$ & $\mathrm{M}(\mathrm{SD})$ & $\begin{array}{l}\text { Non- } \\
\text { standard- } \\
\text { ized B }\end{array}$ & $p$ \\
\hline Clinician-CGI-S & 77 & $4.9(0.8)$ & $3.5(1.3)$ & -1.4 & $<0.0001$ & $3.1(1.3)$ & -1.9 & $<0.0001$ \\
\hline Clinician-CGAS & 76 & $41.2(11.1)$ & $52.3(12.4)$ & 10.9 & $<0.0001$ & $56.6(14.1)$ & 14.8 & $<0.0001$ \\
\hline Clinician-C-SSRS: suicidal ideation & 77 & $1.6(1.8)$ & $0.2(0.6)$ & -1.4 & $<0.0001$ & $0.1(0.5)$ & -1.5 & $<0.0001$ \\
\hline Clinician-C-SSRS: suicidal behavior & 76 & $0.8(1.4)$ & $0.0(0.0)$ & -0.8 & $<0.001$ & $0.1(0.2)$ & -0.7 & .0008 \\
\hline Parent-CDI & 66 & $22.2(7.5)$ & $16.8(8.1)$ & -5.9 & $<0.0001$ & $15.5(5.8)$ & -6.3 & 0.002 \\
\hline Parent-STAI S & 66 & $48.6(11.9)$ & $40.0(12.9)$ & -8.7 & 0.001 & $37.4(11.1)$ & -10.3 & 0.005 \\
\hline Parent-STAI T & 66 & $47.1(10.8)$ & $44.8(9.7)$ & -2.4 & 0.35 & 47.7 (11.9) & 1.6 & 0.67 \\
\hline Child-CDI & 58 & $22.5(8.8)$ & $18.2(8.5)$ & -4.1 & 0.042 & $20.9(9.1)$ & -3.3 & 0.23 \\
\hline Child-STAI C & 48 & $44.0(8.3)$ & $40.8(9.3)$ & -3.6 & 0.024 & $43.2(7.5)$ & -3.0 & 0.18 \\
\hline
\end{tabular}

Bold indicates significant

CGI-S Clinical Global Impression-Severity, CGAS Children's Global Assessment Scale, C-SSRS Columbia-Suicide Severity Rating Scale, CDI Children's Depression Inventory, STAI State-Trait Anxiety Inventory, STAI-S STAI-State, STAI-T STAI-Trait, STAI-C STAI-Children

not require further psychotherapy at the 1-year follow-up. We also found significant improvement in the patients' symptoms that were mostly maintained at 3-6 months after CI treatment ended based upon clinician assessment, and parental and patient reports.

In recent years, accumulating research in children and adolescents support the utilization of models of intervention during crisis that are implemented early and prevent the escalation of mental health issues through community-based approaches [23]. However, most of the recently published brief interventions are aimed at specific psychiatric emergencies, such as suicidality $[8,24]$ and trauma exposure $[25$, 26]. Others focus exclusively on youth in acute crisis and are not for younger children [27]. To the best of our knowledge our model is unique as it addresses a variety of common emergencies in children and adolescents and does not exclude complicated cases with psychiatric comorbidities.

The theoretical and empirical rationale for the psychiatric CI described in this manuscript, is based on integrating the framework of the crisis intervention model [28, 29], with fundamental principles of evidence-based interventions for children and youth psychiatric emergencies. Specifically, CI principles of a family-focused, strength-based approach, are comprehensively incorporated starting at the first stage of the initial psychiatric assessment. Then, an effort is made to identify the principal problem, not only by inquiring about the precipitating event for the crisis (the proverbial "last straw") but also prioritizing problems in terms of which to work on in the short intervention offered. After the focus of the intervention is defined, a suitable protocol is selected, choosing essential and suitable tools according to the case formulation. For example, a case of severe violent outbursts in a 9-year-old diagnosed with obsessive compulsive disorder and attention deficit/hyperactivity disorder. In this child the major problem was defined as the violent outbursts which are secondary to his continuous demand for parents' cooperation with his obsessive rituals. Principles from the SPACE [10] intervention were implemented, with particular emphasis on minimizing parental accommodating behaviors and resisting his demands nonviolently.

The finding that $72 \%$ of children did not require further psychotherapy 1 year following the intervention, suggests that it is a beneficial strategy that is likely to work well within a 'stepped care' model in routine practice. Hence, children with acute crises can be treated immediately with a short treatment that may prevent "stepping up" to treatments of higher intensity. Roberts et al. stated that "Crisis intervention provides a challenge, an opportunity and a turning point within an individual's life" [29]. We believe that when a family which is struggling with their child's acute crisis is offered immediate professional help, the motivation for treatment and the hope for change are high. In this time frame, it is possible that the therapeutic alliance, central in the success of a therapeutic intervention [30], is built rapidly and perhaps assists in achieving treatment goals in such a short intervention. Therefore, we suggest that the relatively low drop-out rate seen in this study is related to the short interval between the crises and the initiation of treatment.

This study has several imitations: first, this is a single center study with a small sample size; lacking a control group. Furthermore, not all cases referred to an initial evaluation were well documented and not all cases that had been treated in the CI unit were included in the final sample due to incomplete data. However, we feel that this setting still 
allows presenting a pragmatic model. In addition, it is possible that unmeasured characteristics affected individuals' willingness to comply with the treatment/experimental protocol, thereby biasing our results [31]. For example, patients who completed the treatment protocol may have been more motivated participants, who adhered more closely to the recommended treatment. Family disorganization may also be an artifact that affects dropout from treatment; children or parents who were not able to attend the treatment sessions on a regular basis were more likely to drop out, therefore, creating a survivorship bias.

In conclusion, we describe a novel intervention for psychiatric emergencies of children and adolescents. We demonstrate this intervention to be feasible and safe and that its implementation significantly shortened waitlist times. The relatively low treatment drop-out rates indicate that the intervention is acceptable. However, future research might add more dimensions to the issue of acceptability using satisfaction questionnaires. We are in the process of expanding the CI unit, focusing on the direct transition from the pediatric ED to the CI unit, and thereby increasing the treatment's accessibility. The necessity for such services is even more vital during these times of emerging unprecedented mental health challenges posed by the COVID-19 pandemic.

Supplementary Information The online version contains supplementary material available at https://doi.org/10.1007/s00787-021-01896-2.

Author contributions All authors contributed to, reviewed, and approved the final manuscript. ID study conceptualization and design, clinical evaluation of the participants, administration of the crisis intervention unit, data curation and interpretation, writing the final manuscript. NH-P study conceptualization and design, data curation and interpretation, data administration, data analysis, writing the final manuscript. SD-I study conceptualization and design, conducting therapeutic sessions with the participants, data curation and interpretation, writing the final manuscript. MR-D conducting therapeutic sessions with the participants, data curation and interpretation, data administration. DG study conceptualization and design, clinical evaluation of the participants, reviewing and editing of the final manuscript. IMP supervising, reviewing and writing the final manuscript.

Funding This study was supported by grants from Foundation Dora and Kirsh Foundation. The funding source had no role in the study design, collection, analysis, or interpretation of data, the writing of the article, or decision to submit the article for publication.

Availability of data and materials The data that support our findings are available upon request from the corresponding author. The data are not publicly available due to privacy or ethical restrictions. Data collected for this study includes individual participants' data. Data cannot be publicly accessible due to Institutional Review Board guidelines. We are open to collaborations with other researchers upon contacting us.

\section{Declarations}

Conflict of interest The authors declare that the research was conducted in the absence of any commercial or financial relationships that could be construed as a potential conflict of interest.

Ethics approval This is a retrospective analysis of prospectively collected data. The screening questionnaires were part of the routine assessment of the patients and their parents. The data were coded anonymously prior to the analysis. The Sheba Medical Center Institutional Review Board approved the study and the need for informed consent was waived due to the retrospective nature of the study.

\section{References}

1. Carubia B, Becker A, Levine BH (2016) Child psychiatric emergencies: updates on trends, clinical care, and practice challenges. Curr Psychiatry Rep 18:41

2. Sheppard R, Deane FP, Ciarrochi J (2018) Unmet need for professional mental health care among adolescents with high psychological distress. Aust N Z J Psychiatry 52:59-67

3. Mapelli E, Black T, Doan Q (2015) Trends in pediatric emergency department utilization for mental health-related visits. J Pediatr 167:905-910

4. Kalb LG, Stapp EK, Ballard ED, Holingue C, Keefer A, Riley A (2019) Trends in psychiatric emergency department visits among youth and young adults in the US. Pediatrics 143(4)

5. Aviram U (2010) Promises and pitfalls on the road to a mental health reform in Israel. Isr J Psychiatry Relat Sci 47:171-183 (discussion 183-194)

6. Allen M FP, Zealberg J, Courier G (2002) Report and recommendations regarding psychiatric emergency and crisis services. American Psychiatric Association, Washington, DC

7. Ribbers A, Sheridan D, Jetmalani A, Magers J, Lin AL, Marshall R (2020) The crisis and transition services (CATS) model: a program to divert youths in mental health crisis from the Emergency Department. Psychiatr Serv 71:1203-1206

8. Haruvi Catalan L, Levis Frenk M, Adini Spigelman E, Engelberg Y, Barzilay S, Mufson L, Apter A, Benaroya Milshtein N, Fennig S, Klomek AB (2020) Ultra-Brief Crisis IPT-A based intervention for suicidal children and adolescents (IPT-A-SCI) pilot study results. Front Psychiatry 11:553422

9. Kendall PCHK (2006) Cognitive-behavioral therapy for anxious children: therapist manual. Workbook Publishing, Ardmore

10. Lebowitz ER, Shimshoni Y (2018) The SPACE program, a parent-based treatment for childhood and adolescent OCD: The case of Jasmine. Bull Menninger Clin 82:266-287

11. DA Brent PK, Goldstein TR (2011) Treating depressed and suicidal adolescents: a clinician's guide. The Guilford Press, New York

12. Weinblatt U, Omer H (2008) Nonviolent resistance: a treatment for parents of children with acute behavior problems. J Marital Fam Ther 34:75-92

13. Mann J (1973) Time-limited psychotherapy. Harvard University Press, Cambridge

14. Shefler G (2000) Time-limited psychotherapy with adolescents. J Psychother Pract Res 9:88-99

15. Kovacs M (1985) The Children's Depression, Inventory (CDI). Psychopharmacol Bull 21:995-998

16. Spielberger C (1973) Manual for the state-trait anxiety inventory for children. Consulting Psychologists Press, Palo Alto 
17. Guy W (1976) Clinical Global Impression. National Institute of Mental Health, Rockville

18. Posner K, Brown GK, Stanley B, Brent DA, Yershova KV, Oquendo MA, Currier GW, Melvin GA, Greenhill L, Shen S, Mann JJ (2011) The Columbia-Suicide Severity Rating Scale: initial validity and internal consistency findings from three multisite studies with adolescents and adults. Am J Psychiatry 168:1266-1277

19. Shaffer D, Gould MS, Brasic J, Ambrosini P, Fisher P, Bird H, Aluwahlia S (1983) A Children's Global Assessment Scale (CGAS). Arch Gen Psychiatry 40:1228-1231

20. Kuznetsova A, Brockhoff PB, Christensen RH (2017) lmerTest package: tests in linear mixed effects models. J Stat Softw 82:1-26

21. Benjamini Y, Hochberg Y (1995) Controlling the false discovery rate: a practical and powerful approach to multiple testing. J R Stat Soc Ser B Methodol 57:289-300

22. Benarous X, Milhiet V, Oppetit A, Viaux S, El Kamel NM, Guinchat V, Guile JM, Cohen D (2019) Changes in the use of emergency care for the youth with mental health problems over decades: a repeated cross sectional study. Front Psychiatry 10:26

23. Vusio F, Thompson A, Birchwood M, Clarke L (2020) Experiences and satisfaction of children, young people and their parents with alternative mental health models to inpatient settings: a systematic review. Eur Child Adolesc Psychiatry 29:1621-1633

24. Morken IS, Dahlgren A, Lunde I, Toven S (2019) The effects of interventions preventing self-harm and suicide in children and adolescents: an overview of systematic reviews. F1000Res 8:890
25. Coldiron ME, Llosa AE, Roederer T, Casas G, Moro MR (2013) Brief mental health interventions in conflict and emergency settings: an overview of four Medecins Sans Frontieres-France programs. Confl Health 7:23

26. Berkowitz SJ, Stover CS, Marans SR (2011) The Child and Family Traumatic Stress Intervention: secondary prevention for youth at risk of developing PTSD. J Child Psychol Psychiatry 52:676-685

27. Henggeler SW, Rowland MD, Halliday-Boykins C, Sheidow AJ, Ward DM, Randall J, Pickrel SG, Cunningham PB, Edwards J (2003) One-year follow-up of multisystemic therapy as an alternative to the hospitalization of youths in psychiatric crisis. J Am Acad Child Adolesc Psychiatry 42:543-551

28. Roberts A, Ottens A (2005) The seven-stage crisis intervention model: a road map to goal attainment, problem solving, and crisis resolution. Brief Treat Crisis Interv 5:329-339

29. Roberts A (2005) Crisis intervention handbook: assessment, treatment, and research. Oxford University Press, Oxford

30. Zilcha-Mano S (2017) Is the alliance really therapeutic? Revisiting this question in light of recent methodological advances. Am Psychol 72:311-325

31. McGowan HM, Nix RL, Murphy SA, Bierman KL, Conduct Problems Prevention Research G (2010) Investigating the impact of selection bias in dose-response analyses of preventive interventions. Prev Sci 11:239-251 